\title{
Development and validation of nomogram based on a novel platelet index score to predict prognosis in patients with renal cell carcinoma
}

\author{
Ruotao Xiao, Yanchun Qin, Lei Liu, Zhigang Chen, Bin Yang, Chuxiao Xu, Wei He, Cheng Liu, Lulin Ma \\ Department of Urology, Peking University Third Hospital, Beijing, China \\ $\square$ Corresponding author: Lulin Ma. Department of Urology, Peking University Third Hospital. E-mail: malulinpku@163.com \\ (c) The author(s). This is an open access article distributed under the terms of the Creative Commons Attribution License (https://creativecommons.org/licenses/by/4.0/). \\ See http://ivyspring.com/terms for full terms and conditions.
}

Received: 2021.03.09; Accepted: 2021.08.18; Published: 2021.08.28

\begin{abstract}
Purpose: This study aims to develop and validate a nomogram based on a novel platelet index score (PIS) to predict prognosis in patients with renal cell carcinoma (RCC).

Patients and methods: We retrospectively analyzed the data of 759 consecutive patients with RCC. The Kaplan-Meier curves were performed to analyze the platelet parameters and PIS was established. The patients were randomly divided into training $(\mathrm{N}=456,60 \%)$ and validation cohorts $(\mathrm{N}=303,40 \%)$. The nomogram was created based on the factors determined by multivariable Cox proportional hazard regression of the training cohort. We assessed the discrimination and calibration of our nomogram in both training and validation cohorts. And then the nomogram was compared with other reported models.
\end{abstract}

Results: High platelet count (PLT>285×109/L) and low platelet distribution width (PDW $\leq 10.95 \mathrm{fL}$ ) were associated with shorter progression-free survival (PFS). Thus, PLT and PDW were incorporated in a novel score system called PIS. On multivariable analysis of training cohort, PIS, American Joint Committee on Cancer $(A J C C)$ stage, and sarcomatoid differentiation were independent prognostic factors, which were all selected into the nomogram. The nomogram exhibited good discrimination in both training (C-index: 0.835$)$ and validation cohorts (C-index: 0.883 ). The calibration curves also showed good agreement between prediction and observation in both cohorts. The $\mathrm{C}$-index of the nomogram (C-index: 0.810 0.902) for predicting 2-year, 3-year, and 4-year PFS were significantly higher than Leibovich (C-index: 0.772 0.813), SSIGN (C-index: 0.775 0.876), Cindolo (C-index: 0.642 0.798), Yaycioglu (C-index: 0.648 0.804), MSKCC (C-index: 0.761 0.862), Karakiewicz (C-index: 0.747 0.851), and AJCC stage models (C-index: 0.759 0.864).

Conclusion: The nomogram based on a novel PIS could offer better risk stratification in patients with RCC.

Key words: nomogram, platelet count, platelet distribution width, platelet index score, renal cell carcinoma, progression-free survival

\section{Introduction}

Renal cell carcinoma (RCC) is the third most common genitourinary worldwide, accounting for $3 \% \sim 5 \%$ of the malignancies in adults [1]. Localize RCC can be cured by surgery, with a favorable 5-year overall survival of $71 \% \sim 88 \%$ [2]. However, even with a relatively good prognosis, approximately $28 \%$ of patients will experience recurrence after curative surgery [3]. Nowadays several prognostic models like
SSIGN, Leibovich, Cindolo, Yaycioglu, MSKCC, and Karakiewicz models have been used in predicting survival for patients with RCC. The reported concordance index (C-index) of the above models were range from 0.67 to $0.84[3-8]$. However, in a prospective external validation cohort, the predictive ability was decreased with a C-index range from 0.587 to 0.69[9]. Thus, it is still worthy for us to further 
explore the potential prognostic factors and develop more accurate predictive models for RCC patients.

Platelets have been recognized as the key factor in hemostasis and wound healing. Recent evidence demonstrated that cancer cells can activate platelets and induce aggregation, which in turn contribute to cancer genesis and metastasis [10]. Platelet count (PLT) is the most notable index that can be affected by the equilibrium between production and consumption. High PLT has been reported as an unfavorable prognostic factor in many malignancies including RCC [11-14]. Recently, mean platelet volume (MPV) and platelet distribution width (PDW), which reflect the size and uniformity of platelets, have shown their prognostic value in thyroid, lung, gastric, and breast cancers [15-18].

To our knowledge, there are few studies that explore the prognostic value of MPV and PDW in RCC populations. Besides, previous models contain similar variables like ECOG-PS, TNM stage, Fuhrman grade, symptom, necrosis, tumor size, et al. [3-8]. However, none of these prognostic models have included any platelet parameters, except the International Metastatic Renal Cell Carcinoma Database Consortium model (IMDC) which was included PLT to predict survival of metastatic RCC[19]. Thus, our study aims were as follows: (1) To explore the prognostic value of PLT, MPV, and PDW in RCC patients and develop a novel platelet index score (PIS); (2) To develop and validate nomogram based on the PIS in our training and validation cohorts; (3) To compare the predictive ability of our nomogram with other reported prognostic models.

\section{Material and methods}

\section{Patients selection}

The clinicopathologic data of renal mass admitted to Peking University Third Hospital from January 2015 to December 2017 were retrospectively analyzed. Inclusion criteria were patients who underwent nephrectomy in our department and pathologically confirmed RCC. Exclusion criteria were as follows: (1) recurrent or bilateral RCC; (2) combined with other malignancies; (3) those with the hematological disease, inflammatory disease, autoimmune disease; (4) those who underwent splenectomy in the past history; (5) those using antiplatelet drugs withing a week before blood collection; (6) missing platelet data. The flow chart was shown in Figure 1. Finally, the eligible study cohort consisted of 759 consecutive RCC patients were further analyzed.

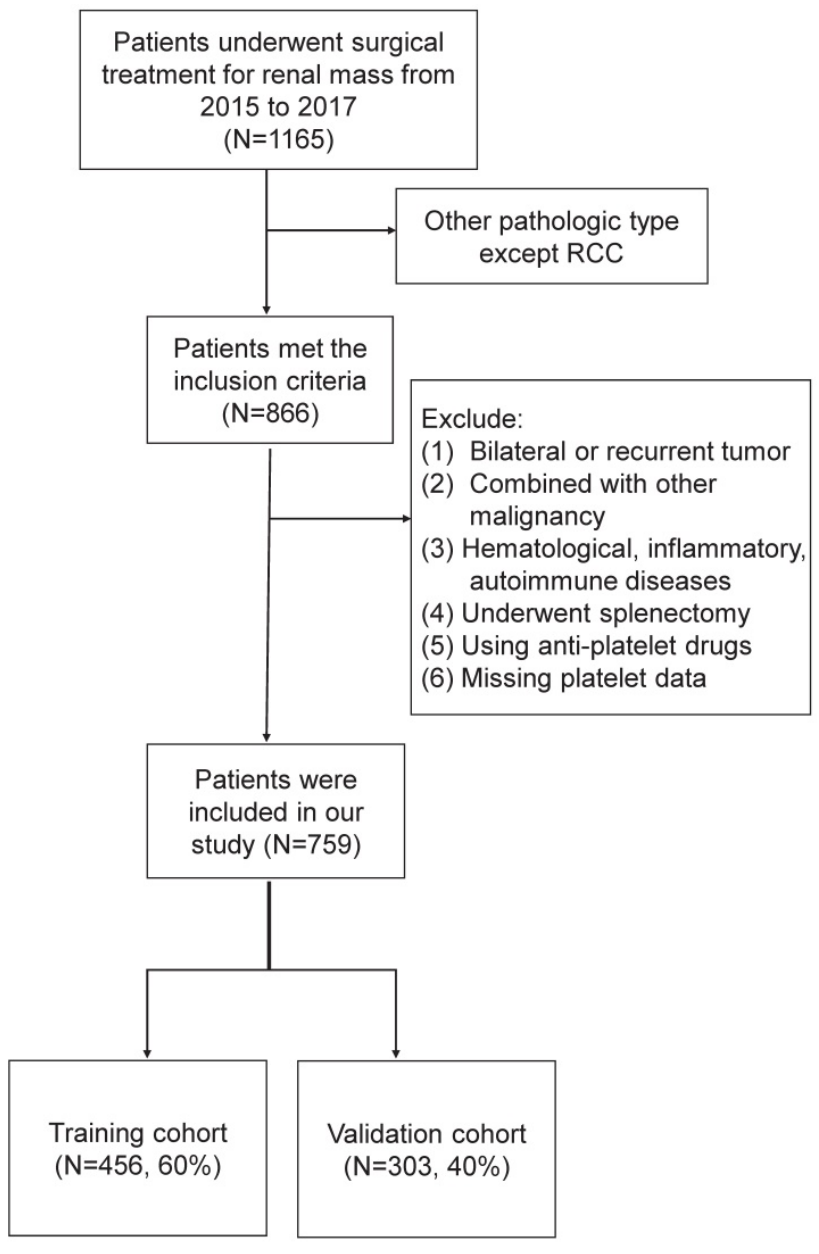

Figure 1. The flow chart of our study cohort.

\section{Data collection}

All patients underwent a blood tests and image examinations. Blood collection was performed within a week before surgery. The whole blood sample was collected in EDTA-containing tubes and processed within an hour after collection. All patients underwent chest X-ray, B-mode ultrasonography, abdominal computed tomography (CT), and/or magnetic resonance imaging (MRI) before surgery. Position emission CT, chest CT, cranial MRI, and bone scans were performed when suspicious distant metastasis. Surgical type and approach were determined by the surgeon' preference based on the current guidelines. All tumor specimen was evaluated by two experienced pathologists. Patients with distant metastasis were treated using tyrosine kinase (TKI) inhibitors as the first-line adjuvant target therapy.

Clinicopathologic variables were collected, including age, sex, body mass index (BMI), symptom at presentation, comorbidities, surgical type, surgical approach, surgical time, interoperative blood loss, tumor size, tumor side, tumor stage, histologic subtype, nuclear grade, necrosis, sarcomatoid and 
rheumatoid differentiation as well as platelet parameters including PLT, MPV, and PDW through electronic medical records of Peking University Third Hospital.

Surgical type, approach, surgical time, and interoperative blood loss were determined based on surgical records. Tumor size, tumor side were evaluated according to the preoperative CT/MRI image report. The tumor stage was evaluated according to both preoperative image and postoperative pathology report, and defined based on the 2016 WHO TNM classification [20]. Nuclear grades were defined according to the 2016 WHO/ISUP grading system [20].

\section{Follow up}

Patients were recommended to follow up regularly at our institution for every 3 months during the first year, every 6 months for the following year, and then annually. Blood tests and chest $\mathrm{x}$-ray were performed at each follow-up while abdominal CT scan was done annually. Follow-up data were collected through medical records or telephone calls. Disease progression was defined as any evidence of recurrence or metastasis, or tumor progress in an already existed metastatic site. Progression-free survival (PFS) was defined as the time from the date of surgery to disease progression.

\section{Statistical analysis}

Normally distributed continuous variable was reported as mean \pm standard deviations. Non-normally distributed continuous variables were reported as medians and interquartile range (IQR). Categorical variables using the frequency count and percentage. The analysis of variance (ANOVA) test or Kruskal-Wallis test were applied to compare continuous variables. The Chi-square test or Fisher exact test was applied to compare categorical variables. The receiver operating characteristic curve (ROC) was used to determine the optimal cut-off value of PLT, MPV, PDW according to survival outcome. The Kaplan-Meier method was performed to analyze the platelet parameters, and the difference between groups was compared using the log-rank test.

To develop a reliable and well-calibrated nomogram, patients were randomly divided into training cohorts $(\mathrm{N}=456,60 \%)$ and validation cohorts $(\mathrm{N}=303,40 \%)$. Univariable and multivariable Cox proportional hazard regression were performed to identify the independent prognostic factors in the training cohort. Selected independent factors were incorporated in the nomogram to predict the probability of 2-year, 3-year, and 4-year PFS. The discrimination of the nomogram was measured by C-index, which ranges from 0.5 (no predictive power) to 1 (perfect prediction) [21]. Calibration was evaluated using calibration curves, which assessed between the observed outcome probabilities and the nomogram predicted probabilities with a bootstrapped resample of 1000 times. The total points of each patient in the validation cohort were calculated according to the nomogram, then Cox regression was performed using the total points as a factor, and finally, the C-index and calibration curve was derived based on the regression analysis. Time-dependent ROC curves and areas under the curves (AUCs) at 2-year, 3-year, and 4-year were generated to assess prognostic accuracy of the nomogram in training and validation cohorts [22].

Improvement in the predictive accuracy was assessed by calculating the integrated discrimination improvement (IDI) and the net reclassification improvement (NRI) [23]. Higher IDI and NRI indicated greater risk discrimination and improved classification. Furthermore, the linear predictor was calculated as the sum of the product of regression coefficients and variables reported by other models, and C-index was calculated according to the linear predictor and then compared with our nomogram. Nomogram establishment and calibration were conducted by $\mathrm{R}$ software (Version 4.0.3) using the "rms" package, and other statistical analysis was performed with SPSS (Version 26, IBM, Armonk, NY) and GraphPad Prism (Version 8, GraphPad Software Inc., San Diego, CA, USA). All tests were on two sided, and $\mathrm{P}<0.05$ was considered statistically significant.

\section{Results}

\section{Study cohort and platelet index score establishment}

Included in the analysis were 759 patients (Table 1). The characteristics of training and validation cohorts were similar. The detailed profile of PLT, MPV, and PDW were shown in Supplement 1. The range of PLT, MPV, and PDW was $57 \sim 689 \times 10^{9} / \mathrm{L}$, 6.2 20.5fL, and 7.9 23.1fL, respectively. Besides, the mean PLT, MPV, and PDW was $223.45 \pm 69.31 \times 10^{9} / \mathrm{L}$, $10.01 \pm 1.4 \mathrm{fL}$, and $13.57 \pm 2.78 \mathrm{fL}$ respectively. Of 665 (87.6\%) patients were followed up with the median time of 36 months (IQR: 31 41). Of 126 patients $(16.6 \%)$ reported disease progression with a median PFS of 57 months (IQR: 43 Not reached). The optimal cut-off values of PLT, MPV, and PDW were $285 \times 10^{9} / \mathrm{L}, 9.45 \mathrm{fL}, 10.95 \mathrm{fL}$ respectively, with the maximal Youden index on ROC analysis (Supplement 2). Then dichotomization of PLT, MPV, 
and PDW was performed according to the cut-off value. Kaplan-Meier curves show high PLT $(\mathrm{P}<0.001)$ and low PDW $(\mathrm{P}<0.001)$ were significantly associated with shorter PFS. While MPV $(\mathrm{P}=0.141)$ was not significantly associated with PFS in our study cohort (Fig. 2).

Table 1. Demographics and clinicopathologic characteristics of patients with renal cell carcinoma

\begin{tabular}{lllll}
\hline Variable & $\begin{array}{l}\text { Study cohort } \\
(\mathrm{N}=759)\end{array}$ & $\begin{array}{l}\text { Training cohort } \\
(\mathrm{N}=456)\end{array}$ & $\begin{array}{l}\text { Validation cohort } \\
(\mathrm{N}=303)\end{array}$ & $\mathrm{P}$ \\
\hline $\begin{array}{l}\text { Age, years } \\
\text { Sex }\end{array}$ & $56.47 \pm 12.03$ & $56.56 \pm 11.98$ & $56.33 \pm 12.12$ & 0.793 \\
$\begin{array}{l}\text { Male } \\
\text { Female }\end{array}$ & $523(68.9)$ & $312(68.4)$ & $211(69.6)$ & 0.749 \\
$\begin{array}{l}\text { Symptom at } \\
\text { presentation }\end{array}$ & $236(31.1)$ & $144(31.6)$ & $92(30.4)$ & 0.792 \\
$\begin{array}{l}\text { BMI, kg/m } \\
\text { Comorbidities }\end{array}$ & $25.15 \pm 3.41$ & $25.21 \pm 3.55$ & $25.06 \pm 3.19$ & 0.552 \\
$\begin{array}{l}\text { Hypertension } \\
\text { Coronary heart }\end{array}$ & $300(39.5)$ & $190(41.7)$ & $110(36.3)$ & 0.150 \\
disease & $63(8.3)$ & $37(8.1)$ & $26(8.6)$ & 0.893 \\
Diabetes mellitus & $117(15.4)$ & $68(14.9)$ & $49(16.2)$ & 0.608 \\
$\begin{array}{l}\text { Surgical type } \\
\text { NSS }\end{array}$ & $378(49.8)$ & $223(48.9)$ & $155(51.2)$ & 0.554 \\
RN & $381(50.2)$ & $233(51.1)$ & $148(48.8)$ & \\
Surgical approach & & & & \\
$\quad \begin{array}{l}\text { Open } \\
\text { Laparoscopic }\end{array}$ & $338(44.5)$ & $208(45.6)$ & $130(42.9)$ & 0.502 \\
$\begin{array}{l}\text { Tumor size, cm } \\
\text { Tumor side }\end{array}$ & $421(55.5)$ & $248(54.4)$ & $173(57.1)$ & \\
Left & $4.89 \pm 3.01$ & $4.93 \pm 3.1$ & $4.83 \pm 2.87$ & 0.643 \\
Right & $362(47.7)$ & $215(47.1)$ & $147(48.5)$ & 0.767 \\
$\begin{array}{l}\text { AJCC tumor stage } \\
\text { I }\end{array}$ & $397(52.3)$ & $241(52.9)$ & $156(51.5)$ & \\
II & $465(61.3)$ & $277(60.7)$ & $188(62)$ & \\
\hline
\end{tabular}

A

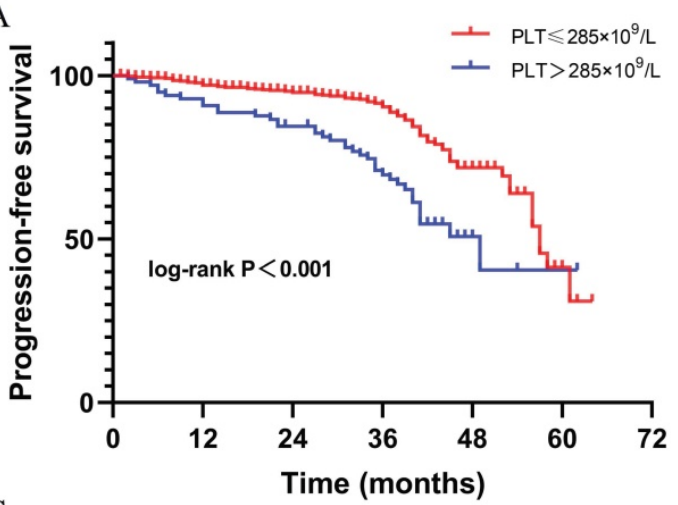

$\mathrm{C}$

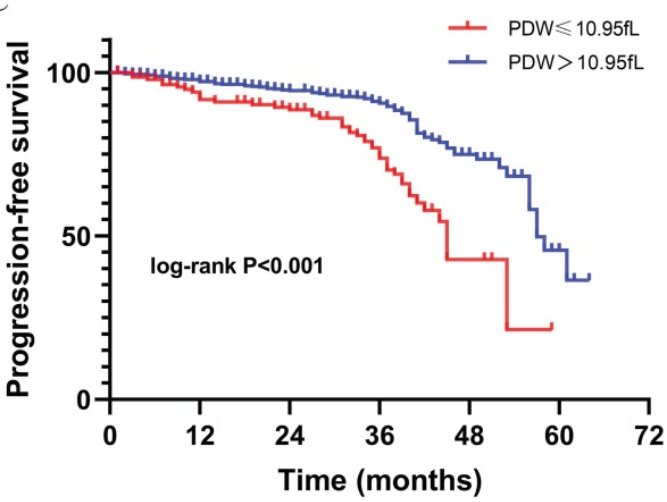

\begin{tabular}{|c|c|c|c|c|}
\hline Variable & $\begin{array}{l}\text { Study cohort } \\
(\mathrm{N}=759)\end{array}$ & $\begin{array}{l}\text { Training cohort } \\
(\mathrm{N}=456)\end{array}$ & $\begin{array}{l}\text { Validation cohort } \\
(\mathrm{N}=303)\end{array}$ & $\mathrm{P}$ \\
\hline III & $206(27.1)$ & $121(26.5)$ & $85(28.1)$ & \\
\hline IV & $64(8.4)$ & $41(9.0)$ & $23(7.6)$ & \\
\hline \multicolumn{5}{|l|}{ Histologic subtype } \\
\hline CCRCC & $656(86.4)$ & $398(87.3)$ & $258(85.1)$ & 0.449 \\
\hline Non-CCRCC & 103 (13.6) & $58(12.7)$ & 45 (14.9) & \\
\hline \multicolumn{5}{|l|}{ Nuclear grade } \\
\hline I & $90(11.9)$ & $54(11.8)$ & 36 (11.9) & 0.548 \\
\hline II & $442(58.2)$ & $263(57.7)$ & $179(59.1)$ & \\
\hline III & $142(18.7)$ & $93(20.4)$ & $49(16.2)$ & \\
\hline IV & $39(5.1)$ & $21(4.6)$ & $18(5.9)$ & \\
\hline Unknow & $46(6.1)$ & $25(5.5)$ & $21(6.9)$ & \\
\hline Necrosis & $129(17)$ & 75 (16.4) & $54(17.8)$ & 0.623 \\
\hline $\begin{array}{l}\text { Sarcomatoid } \\
\text { differentiation }\end{array}$ & $21(2.8)$ & $12(2.6)$ & $9(3)$ & 0.340 \\
\hline $\begin{array}{l}\text { Rheumatoid } \\
\text { differentiation }\end{array}$ & $18(2.4)$ & $11(2.4)$ & $7(2.3)$ & 0.609 \\
\hline \multicolumn{5}{|l|}{ Platelet parameters } \\
\hline PLT,$\times 10^{9} / \mathrm{L}$ & $223.45 \pm 69.31$ & $223.42 \pm 72.45$ & $223.49 \pm 64.4$ & 0.988 \\
\hline $\mathrm{MPV}, \mathrm{fL}$ & $10.01 \pm 1.4$ & $10.06 \pm 1.38$ & $9.93 \pm 1.44$ & 0.184 \\
\hline PDW, fL & $13.57 \pm 2.78$ & $13.59 \pm 2.85$ & $13.53 \pm 2.66$ & 0.747 \\
\hline \multicolumn{5}{|l|}{ PIS score } \\
\hline 0 & $531(70)$ & $323(70.8)$ & $208(68.6)$ & 0.821 \\
\hline 1 & $178(23.5)$ & $104(22.8)$ & $74(24.4)$ & \\
\hline 2 & $50(6.6)$ & $29(6.4)$ & $21(6.9)$ & \\
\hline \multicolumn{5}{|l|}{ Follow-up data } \\
\hline Progression-free & $539(71)$ & $319(70)$ & $220(72.6)$ & 0.571 \\
\hline Progression & $126(16.6)$ & $81(17.8)$ & 45 (14.9) & \\
\hline Loss of follow up & $94(12.4)$ & $56(12.3)$ & 38 (12.5) & \\
\hline \multicolumn{5}{|c|}{$\begin{array}{l}\text { Abbreviations: BMI: Body mass index; NSS: Nephron sparing surgery; RN: Radical } \\
\text { Nephrectomy; AJCC: American Joint Committee on Cancer; CCRCC: Clear cell } \\
\text { renal cell carcinoma; PLT: platelet count; MPV: mean platelet volume; PDW: } \\
\text { platelet distribution width; PIS score: platelet index score. }\end{array}$} \\
\hline
\end{tabular}

B

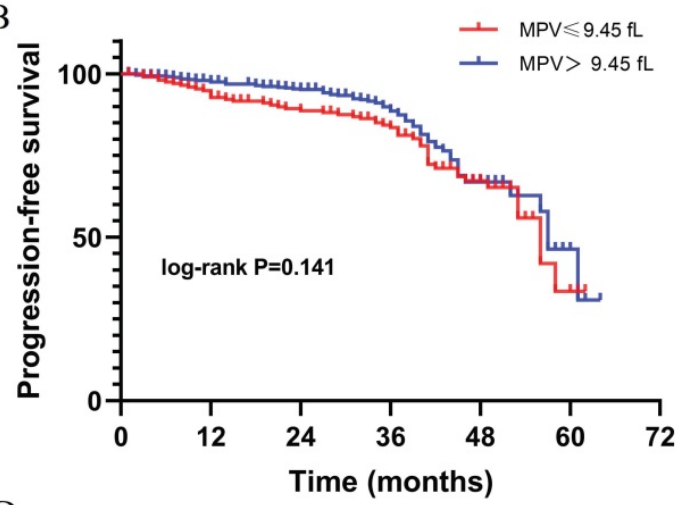

$\mathrm{D}$

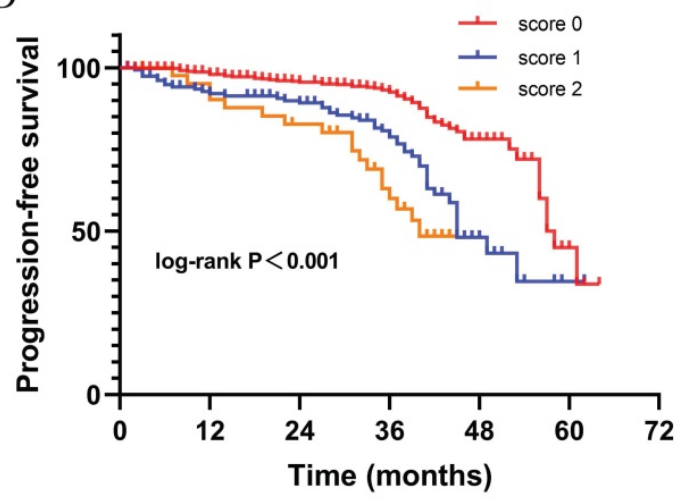

Figure 2. The Kaplan-Meier curves correlated with progression-free survival for platelet parameters. (A) PLT; (B) MPV; (C) PDW; (D) PIS. 


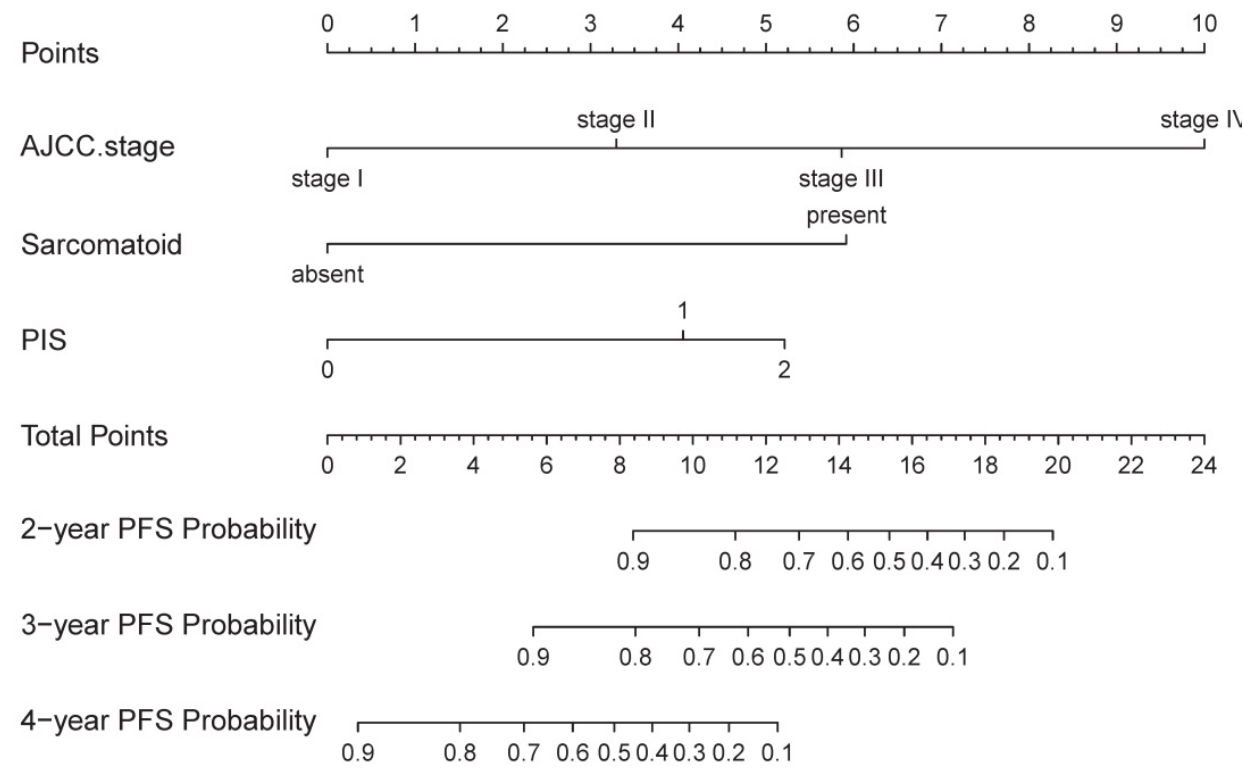

Figure 3. The nomogram was developed in the training cohort, with AJCC Stage, Sarcomatoid differentiation, PIS.

To further investigate the prognostic value of platelet parameters in RCC, we established a platelet index score (PIS) by combining PLT and PDW. The PIS were defined as follows: (1) Score 0: patients with low PLT $\left(\leq 285 \times 10^{9} / \mathrm{L}\right)$ and high PDW (>10.95fL); (2) Score 1: patients with either high PLT $\left(>285 \times 10^{9} / \mathrm{L}\right)$ or low PDW ( $\leq 10.95 \mathrm{fL})$; (3) Score 2: patients with high PLT $\left(>285 \times 10^{9} / \mathrm{L}\right)$ and low PDW $(\leq 10.95 \mathrm{fL})$. In our study cohort, the cases of PIS score $0,1,2$ were 531 $(70 \%), 178(23.5 \%), 50(6.6 \%)$ respectively. The Kaplan-Meier curve shows patients with score 0 have significantly longer PFS than those with score 1 and score $2(\mathrm{P}<0.001$, Fig. 2$)$. Besides, patients with higher PIS associated with lower BMI $(\mathrm{P}=0.002)$, more symptom at presentation $(\mathrm{P}=0.035)$, larger tumor size $(\mathrm{P}<0.001)$, advanced AJCC stage $(\mathrm{P}<0.001)$ and nuclear grade $(\mathrm{P}=0.001)$, more necrosis $(\mathrm{P}=0.004)$, sarcomatoid differentiation $(\mathrm{P}=0.043)$, and rheumatoid differentiation ( $\mathrm{P}=0.001)$ (Table 2).

\section{Nomogram development and validation}

On univariable and multivariable analysis in the training cohort, higher PIS (HR: 2.098 for score 1, 2.775 for score 2, $\mathrm{P}<0.001$ ), higher AJCC stage (HR: 2.746 for II, 5.352 for III, 13.662 for IV, $\mathrm{P}<0.001)$, and sarcomatoid differentiation (HR: 2.032, $\mathrm{P}=0.030$ ) were independent factors predict shorter PFS (Table 3 ). Thus, we established the nomogram by incorporating all the independent factors to predict 2-year, 3-year, and 4-year PFS (Fig. 3). The C-index was 0.835 for the training cohort and the calibration curves for the probability of 2-year, 3-year, and 4-year PFS showed an optimal agreement with the prediction by nomogram and actual observation (Fig. 4). In the validation cohort, the C-index was 0.883 and calibration curves also showed good agreement between prediction and observation (Fig. 4). To further compare the discrimination of the nomogram in training and validation cohorts, the 2-year, 3-year, and 4-year of time-dependent ROC curves were showed in Figure 5, which suggested good discrimination in both training and validation cohorts.

Table 2. The correlation between platelet index score and clinicopathologic characteristics.

\begin{tabular}{lllll}
\hline Variable & \multicolumn{3}{l}{ PIS } & P \\
\cline { 2 - 4 } & $0(\mathrm{~N}=531)$ & $1(\mathrm{~N}=178)$ & $2(\mathrm{~N}=50)$ & \\
\hline Age, year & $56.39 \pm 11.98$ & $57.18 \pm 12.50$ & $54.84 \pm 10.82$ & 0.459 \\
Gender & & & & \\
Male & $369(69.5)$ & $119(66.9)$ & $35(70)$ & 0.793 \\
Female & $162(30.5)$ & $59(33.1)$ & $15(30)$ & \\
Symptom at presentation & $107(20.7)$ & $51(29.7)$ & $18(39.1)$ & 0.002 \\
BMI, kg/m ${ }^{2}$ & $25.34 \pm 3.26$ & $24.81 \pm 3.67$ & $24.26 \pm 3.80$ & 0.035 \\
Tumor size, cm & $4.61 \pm 2.88$ & $5.15 \pm 2.91$ & $7.01 \pm 3.69$ & $<0.001$ \\
AJCC stage & & & & \\
I II & $379(71.4)$ & $90(50.6)$ & $20(40)$ & $<0.001$ \\
III IV & $152(28.6)$ & $88(49.4)$ & $30(60)$ & \\
Histologic subtype & & & & \\
CCRCC & $433(86.6)$ & $147(89.6)$ & $38(84.4)$ & 0.513 \\
Non-CCRCC & $67(13.4)$ & $17(10.4)$ & $7(15.6)$ & \\
Nuclear grade & & & & \\
I-II & $387(78)$ & $118(70.2)$ & $27(56.3)$ & 0.001 \\
III-IV & $109(22)$ & $50(29.8)$ & $21(43.8)$ & \\
Necrosis & $82(15.4)$ & $30(16.9)$ & $17(34)$ & 0.004 \\
Sarcomatoid differentiation & $11(2.1)$ & $6(3.4)$ & $4(8)$ & 0.043 \\
Rheumatoid differentiation & $6(1.1)$ & $8(4.5)$ & $4(8)$ & 0.001 \\
\hline Abbreviations: BMI: Body mass index; AJCC: American Joint Committee on \\
Cancer; CCRCC: Clear cell renal cell carcinoma; PIS score: platelet index score.
\end{tabular}

The NRI and IDI were performed to show the improvement of the nomogram compared to the model without PIS. The NRI were $0.284(\mathrm{P}<0.001)$, $0.384(\mathrm{P}<0.001)$, and $0.307(\mathrm{P}=0.02)$ for 2-year, 3-year, and 4-year PFS respectively, which significantly 
improved compared to the model without PIS. The IDI were $0.056(\mathrm{P}=0.07), 0.076(\mathrm{P}<0.001)$, and 0.08 $(\mathrm{P}=0.03)$ for 2-year, 3-year, and 4-year PFS respectively, which also significantly improved compared to the model without PIS.

\section{Comparison of our nomogram and other models}

Of our study cohort, the C-index of our nomogram was $0.902,0.881,0.810$ respectively in predicting 2-year, 3-year, and 4-year PFS (Table 4). Models of SSIGN [4], Leibovich [3], Cindolo [6], Yaycioglu [5], MSKCC [7], Karakiewicz [8], and conventional AJCC staging system [20] were externally validated in our study cohort. The SSIGN performed the best (C-index: 0.775 0.876) and Cindolo performed the worst (C-index: 0.642 0.798). Besides, our nomogram displayed a better accuracy than Leibovich, SSIGN model, Cindolo, Yaycioglu, MSKCC, Karakiewicz, and AJCC stage models.

Table 3. Univariable and multivariable analysis to identify the independent factors in the training cohort

\begin{tabular}{lllll}
\hline Variable & Univariable & \multicolumn{3}{l}{ Multivariable } \\
\cline { 2 - 5 } & HR $(95 \% \mathrm{CL})$ & $\mathrm{P}$ & $\mathrm{HR}(95 \% \mathrm{CL})$ & $\mathrm{P}$ \\
\hline $\begin{array}{l}\text { Age, year } \\
\text { Symptom at } \\
\text { presentation }\end{array}$ & $1.019(0.999 \sim 1.039)$ & 0.059 & & \\
BMI, kg/m & $2.132(1.332 \sim 3.414)$ & 0.002 & & \\
AJCC stage & $0.890(0.833 \sim 0.950)$ & 0.001 & & \\
I & & & & \\
II & Ref & & Ref & 0.216 \\
III & $2.322(0.529 \sim 10.186)$ & 0.264 & 2.558 & \\
\hline
\end{tabular}

A

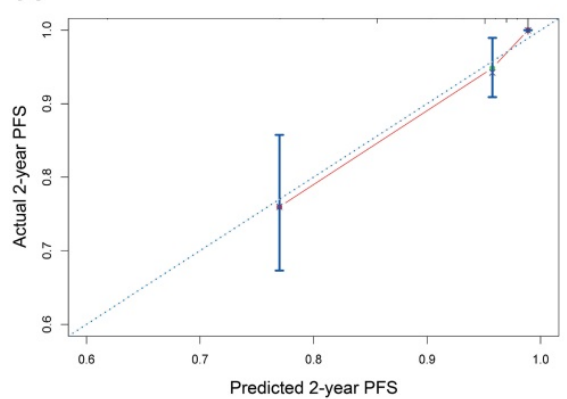

B

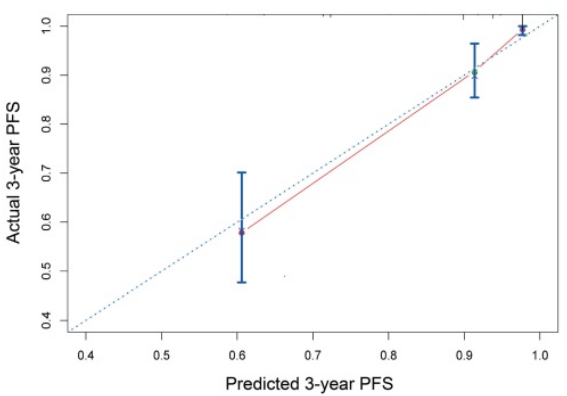

C

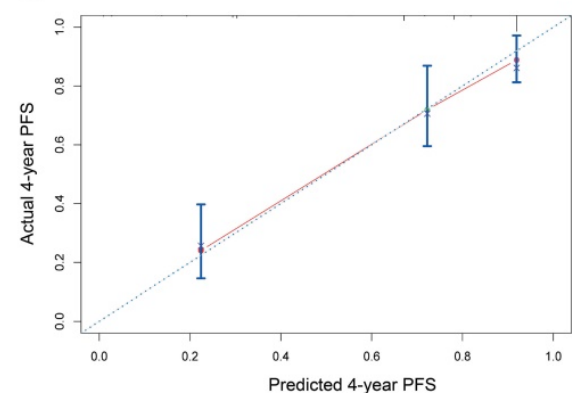

E

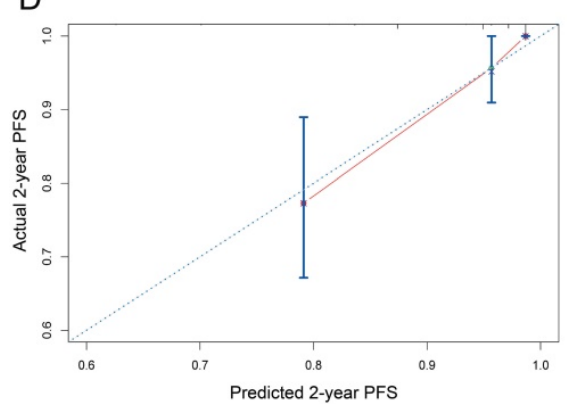

\begin{tabular}{|c|c|c|c|c|}
\hline \multirow[t]{2}{*}{ Variable } & \multicolumn{2}{|l|}{ Univariable } & \multicolumn{2}{|l|}{ Multivariable } \\
\hline & HR (95\%CL) & $\mathrm{P}$ & HR (95\%CL) & $\mathrm{P}$ \\
\hline IV & $\begin{array}{l}16.763 \\
(15.422 \sim 46.052)\end{array}$ & $<0.001$ & $\begin{array}{l}12.664 \\
(6.298 \sim 10.698)\end{array}$ & $<0.001$ \\
\hline $\begin{array}{l}\text { Histologic subtype } \\
\text { (CCRCC vs } \\
\text { Non-CCRCC) }\end{array}$ & 1.107 (0.501 2.442) & 0.802 & & \\
\hline $\begin{array}{l}\text { Nuclear grade (III-IV vs } \\
\text { I-II) }\end{array}$ & $4.077(2.596 \sim 6.404)$ & $<0.001$ & & \\
\hline Necrosis & $4.169(2.668 \sim 6.514)$ & $<0.001$ & & \\
\hline $\begin{array}{l}\text { Sarcomatoid } \\
\text { differentiation }\end{array}$ & $9.222(4.561 \sim 18.648)$ & $<0.001$ & $\begin{array}{l}4.866 \\
(2.213 \sim 10.698)\end{array}$ & $<0.001$ \\
\hline $\begin{array}{l}\text { Rheumatoid } \\
\text { differentiation } \\
\text { PIS }\end{array}$ & 7.646 (3.277 17.839) & $<0.001$ & & \\
\hline 0 & Ref & & Ref & \\
\hline 1 & $2.609(1.616 \sim 4.210)$ & $<0.001$ & 2.577 (1.508 4.403) & 0.001 \\
\hline 2 & $5.569(2.895 \sim 10.715)$ & $<0.001$ & 4.087 (1.980 8.438) & $<0.001$ \\
\hline
\end{tabular}

Table 4. Comparison of our nomogram and other reported models in study cohort

\begin{tabular}{llll}
\hline Models & \multicolumn{2}{l}{ C-index for our study cohort } \\
\cline { 2 - 4 } & 2 -year & 3-year & 4-year \\
\hline Nomogram & 0.902 & 0.881 & 0.810 \\
SSIGN & 0.876 & $0.821^{*}$ & 0.775 \\
Leibovich & $0.813^{*}$ & $0.764^{*}$ & 0.772 \\
Cindolo & $0.798^{*}$ & $0.732^{*}$ & $0.642^{*}$ \\
Yaycioglu & $0.804^{*}$ & $0.737^{*}$ & $0.648^{*}$ \\
MSKCC & $0.862^{*}$ & $0.810^{*}$ & $0.761^{*}$ \\
Karakiewicz & $0.851^{*}$ & $0.793^{*}$ & $0.747^{*}$ \\
AJCC stage & $0.863^{*}$ & $0.837^{*}$ & $0.759^{*}$
\end{tabular}

Notes: *: means the $\mathrm{P}<0.05$ in comparison of $\mathrm{C}$-index between our nomogram and other models.
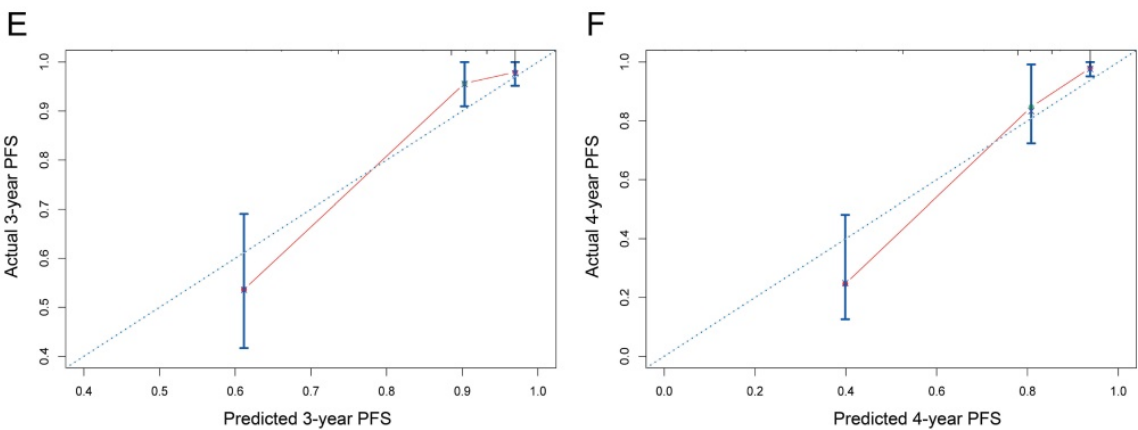

Figure 4. The calibration curves of nomogram to predict progression-free survival in training cohort and validation cohort. (A-C) 2-year, 3-year, and 4-year calibration curves for training cohort; (D-F) 2-year, 3-year, and 4-year calibration curves for validation cohort. 


\section{A}

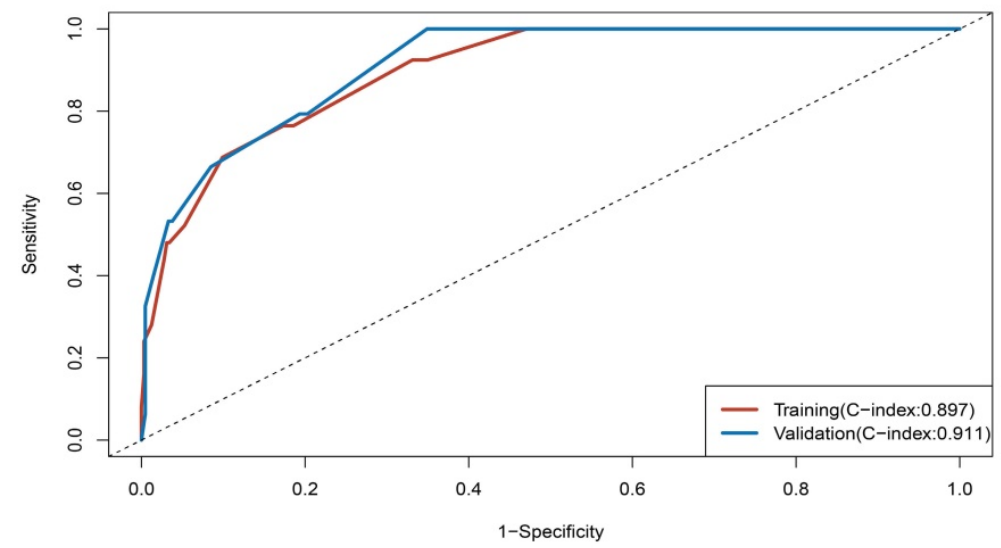

B

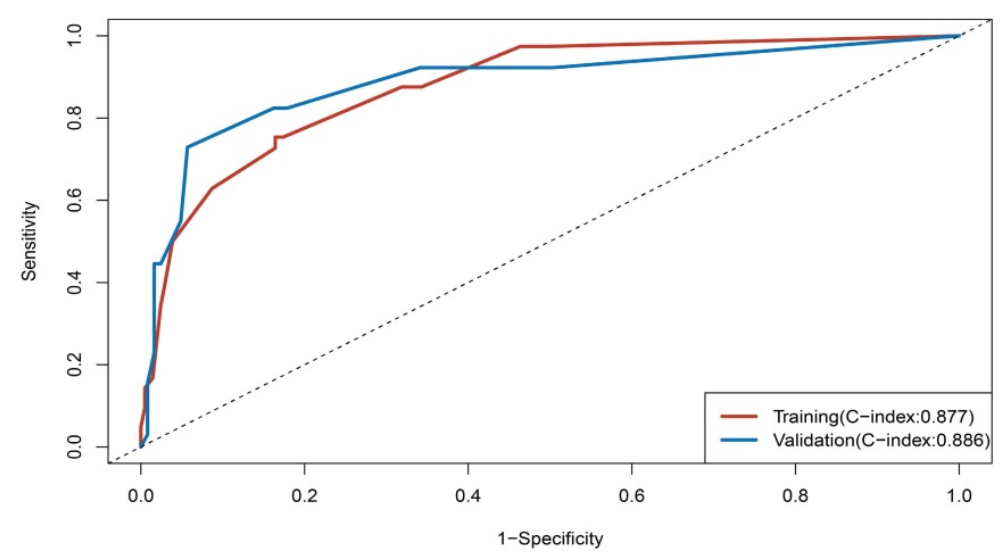

C

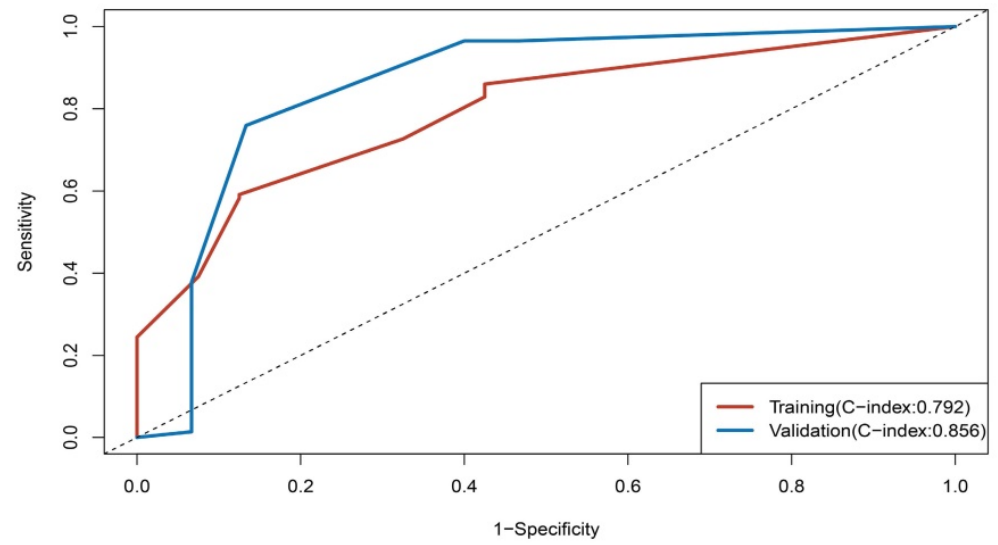

Figure 5. Time-dependent ROC curves and C-index of training and validation cohorts. (A) ROC curve at 2-year; (B) ROC curve at 3-year; (C) ROC curve at 4-year.

\section{Discussion}

The ability to predict oncologic outcomes in RCC patients is essentially significant to clinicians. Platelets parameters have been explored as a promising biomarker in predicting oncologic outcomes in cancers. In this study we analyzed a consecutive 759 RCC patients and reported several noteworthy findings. Firstly, high PLT and low PDW were associated with shorter PFS in our study group. Then we established a novel score system named PIS by combining PLT and PDW, which have shown their significant correlation with disease progression. Secondly, we created a nomogram based on the PIS and other independent factors to predict PFS, which exhibited good discrimination and calibration in the training and validation cohort. Thirdly, our nomogram displayed better accuracy in predicting 
2-year, 3-year, and 4-year PFS than other reported models and conventional AJCC staging system.

Notably, several studies have explored that high PLT was related to worse prognosis in RCC populations, which consistent with our findings [14, 24]. Recent evidence also showed increased PLT had a lower response rate to tyrosine kinase inhibitors therapy and shorter overall survival in metastatic RCC [25]. Thus, PLT was included in the IMDC model to predict prognosis for metastatic RCC patients [19]. However, the conflicting results were also reported that PLT was not an independent factor predicting prognosis after adjusting some known pathologic factors, which suggested we should focus more on other platelet parameters that can better reflect the function rather than platelet count alone [26, 27].

Preclinical studies have found cancer cells can activate platelet through a wide variety of crosstalk, eventually caused tumor genesis and metastasis [8]. MPV and PDW can serve as indicators reflecting the activation of platelets [28, 29]. Clinical studies have found a strong correlation with diagnosis and prognosis in several malignancies [15-18]. Seles $M$ et al. [30] have found MPV represented a highly significant predictor of recurrence and cancer-specific death in patients with RCC. Although our study failed to show the prognostic value of MPV, the PDW was another novel biomarker in predicting prognosis for RCC patients, which has not been investigated before. Several studies have shown high PDW was associated with poor survival in hepatocellular, breast cancers as well as skull base chordoma [31-33]. However, low PDW has also been reported that correlated with worse survival in endometrial, esophageal, gastric cancers [34-36], which consistent with our study result that low PDW was related to shorter PFS in RCC patients. The reason for the conflicting results was unclear. We speculate that within the tumor microenvironment, tumor cells can trigger activated platelet release granules that contain platelet-derived growth factor (PDGF), vascular endothelial growth factor (VEGF), and transforming growth factor $\beta$ (TGF- $\beta$ ), which lead to decreased in size and increased heterogeneity of platelet at an early stage of tumor [10]. But as tumor growth and tumor burden increased, more and more platelets were affected by cancer cells, and eventually, the heterogeneity was decreased. However, the underline mechanism remains to be elucidated.

Furthermore, we created a novel score system called PIS by combining PLT and PDW and found that high PIS correlated with shorter PFS in RCC. Chen $\mathrm{H}$ et al. [36] was the first to established a similar platelet index score by combining PLT, MPV, and PDW, which showed its strong correlation with recurrent-free survival and overall survival in endometrial cancer. It is worth noting that in RCC patients, conventional prognostic models, except the IMDC model, were not included any platelet parameters $[3-8,19]$. Since our study result has shown the prognostic value of PIS, we created a nomogram based on PIS to predict PFS in patients with RCC. The nomogram performed well in the prediction of RCC patients by its reported C-index $(0.835$ and 0.883 for the training and validation cohort) and calibration curve. Although the external validation of other models has also shown its good discrimination with C-index range from 0.642 to 0.895 , the nomogram is more accurate to predict PFS than the models like Leibovich, SSIGN, Cindolo, MSKCC, Karakiewicz, and Yaycioglu models as well as AJCC staging system. Besides, our nomogram only included three features, which was simpler than other models. More importantly, our findings not only raise significant concerns regarding the prognostic value of preoperative platelet parameters in patients with RCC but also predict prognosis for patients with RCC in a more accurate way by combining platelet parameters with conventional prognostic factors.

Several limitations may apply to our findings. We cannot eliminate the inherent bias owing to the retrospective nature of our study. As we know the preoperative drugs, inflammation, and hematologic disease may affect platelet function and parameters. But we could only achieve this information from the electronic medical records, which may not reflect the real state of an individual. Besides, although our study demonstrated that the PDW and PLT were associated with prognosis, the cut-off value of these parameters varied between different studies. Finally, the nomogram was only developed and validated in a retrospective single-center population. Thus, future studies are needed to externally validate the proposed nomogram in a multicenter, prospective, and large cohort.

\section{Conclusion}

In conclusion, we found high PLT, low PDW, and high PIS were associated with shorter PFS in patients with RCC. Then we established a nomogram based on the PIS for predicting PFS in patients with RCC. The nomogram could offer better risk stratification than other reported prognostic models in patients with RCC.

\section{Ethical approval}

The study was conducted in accordance with the Declaration of Helsinki and was approved by the Peking University Third Hospital Medical Science Research Ethics Committee. Because of the 
retrospective nature of the study, patient consent for inclusion was waived.

\section{Supplementary Material}

Supplementary figures.

http://www.jcancer.org/v12p6301s1.pdf

\section{Acknowledgments}

We thank all the patients whose details form the basis of this study. Besides, we thank all the research secretaries who participated in collecting and correcting the clinicopathologic and follow-up data in the study.

\section{Competing Interests}

The authors have declared that no competing interest exists.

\section{References}

1. Siegel RL, Miller KD, Jemal A. Cancer statistics, 2020. CA Cancer J Clin. 2020;70(1):7-30.

2. Leibovich BC, Lohse CM, Crispen PL, et al. Histological subtype is an independent predictor of outcome for patients with renal cell carcinoma. J Urol. 2010;183(4):1309-1315.

3. Leibovich BC, Blute ML, Cheville JC, et al. Prediction of progression after radical nephrectomy for patients with clear cell renal cell carcinoma: a stratification tool for prospective clinical trials. Cancer. 2003;97(7):1663-1671.

4. Frank I, Blute ML, Cheville JC, Lohse CM, Weaver AL, Zincke H. An outcome prediction model for patients with clear cell renal cell carcinoma treated with radical nephrectomy based on tumor stage, size, grade and necrosis: the SSIGN score. J Urol. 2002;168(6):2395-2400.

5. Yaycioglu O, Roberts WW, Chan T, Epstein JI, Marshall FF, Kavoussi LR. Prognostic assessment of nonmetastatic renal cell carcinoma: a clinically based model. Urology. 2001;58(2):141-145.

6. Cindolo L, de la Taille A, Messina G, et al. A preoperative clinical prognostic model for non-metastatic renal cell carcinoma. BJU Int. 2003;92(9):901-905.

7. Sorbellini M, Kattan MW, Snyder ME, et al. A postoperative prognostic nomogram predicting recurrence for patients with conventional clear cell renal cell carcinoma. J Urol. 2005;173(1):48-51.

8. Karakiewicz PI, Briganti A, Chun FK, et al. Multi-institutional validation of a new renal cancer-specific survival nomogram. J Clin Oncol. 2007; 25(11):1316-22.

9. Correa AF, Jegede O, Haas NB, et al. Predicting Renal Cancer Recurrence: Defining Limitations of Existing Prognostic Models With Prospective Trial-Based Validation. J Clin Oncol. 2019;37(23):2062-2071.

10. Haemmerle M, Stone RL, Menter DG, Afshar-Kharghan V, Sood AK. The Platelet Lifeline to Cancer: Challenges and Opportunities. Cancer Cell. 2018:33(6):965-983.

11. Hufnagel DH, Cozzi GD, Crispens MA, Beeghly-Fadiel A. Platelets, Thrombocytosis, and Ovarian Cancer Prognosis: Surveying the Landscape of the Literature. Int J Mol Sci. 2020;21(21): 8169.

12. Qu CH, Li T, Tang ZP, Zhu XR, Han JY, Tian H. Platelet Count is Associated with the Rate of Lymph Node Metastasis in Lung Adenocarcinoma. Cancer Manag Res. 2020;12:9765-9774.

13. Oh SE, Seo JE, An JY, et al. Prognostic Impact of Increased Perioperative Platelet Count in Gastric Cancer Patients. J Surg Res. 2019;242:296-303.

14. Bensalah K, Leray E, Fergelot $P$, et al. Prognostic value of thrombocytosis in renal cell carcinoma. J Urol. 2006;175(3 Pt 1):859-863.

15. Yu YJ, Li N, Yun ZY, et al. Preoperative mean platelet volume and platelet distribution associated with thyroid cancer. Neoplasma. 2017;64(4):594-598.

16. Shen XB, Wang $Y$, Shan BJ, et al. Prognostic Significance Of Platelet-To-Lymphocyte Ratio (PLR) And Mean Platelet Volume (MPV) During Etoposide-Based First-Line Treatment In Small Cell Lung Cancer Patients. Cancer Manag Res. 2019;11:8965-8975.

17. Gu M, Zhai Z, Huang L, et al. Pre-treatment mean platelet volume associates with worse clinicopathologic features and prognosis of patients with invasive breast cancer. Breast Cancer. 2016;23(5):752-760.

18. Shen $\mathrm{XM}, \mathrm{Xia} Y \mathrm{Y}, \mathrm{Lian} \mathrm{L}$, et al. Mean platelet volume provides beneficial diagnostic and prognostic information for patients with resectable gastric cancer. Oncol Lett. 2016;12(4):2501-2506.

19. Heng DY, Xie W, Regan MM, et al. Prognostic factors for overall survival in patients with metastatic renal cell carcinoma treated with vascular endothelial growth factor-targeted agents: results from a large, multicenter study. J Clin Oncol. 2009;27(34):5794-5799.
20. Moch H, Cubilla AL, Humphrey PA, Reuter VE, Ulbright TM. The 2016 WHO Classification of Tumours of the Urinary System and Male Genital Organs-Part A: Renal, Penile, and Testicular Tumours. Eur Urol. 2016;70(1):93-105.

21. Steyerberg EW, Vergouwe Y. Towards better clinical prediction models: seven steps for development and an $\mathrm{ABCD}$ for validation. Eur Heart J. 2014;35(29):1925-1931.

22. Heagerty PJ, Lumley T, Pepe MS. Time-dependent ROC curves for censored survival data and a diagnostic marker. Biometrics. 2000;56(2):337-344.

23. Pencina MJ, D'Agostino RB, Sr., D'Agostino RB, Jr., Vasan RS. Evaluating the added predictive ability of a new marker: from area under the ROC curve to reclassification and beyond. Stat Med. 2008;27(2):157-172; discussion 207-112.

24. Choi JY, Ko YH, Song PH. Clinical significance of preoperative thrombocytosis in patients who underwent radical nephrectomy for nonmetastatic renal cell carcinoma. Investig Clin Urol. 2016;57(5):324-329.

25. Hamilton Z, Lee HJ, Jimenez J, et al. Change in platelet count as a prognostic indicator for response to primary tyrosine kinase inhibitor therapy in metastatic renal cell carcinoma. BJU Int. 2016;118(6):927-934.

26. Karakiewicz PI, Trinh QD, Lam JS, et al. Platelet count and preoperative haemoglobin do not significantly increase the performance of established predictors of renal cell carcinoma-specific mortality. Eur Urol. 2007;52(5):1428-1436

27. Brookman-May S, May M, Ficarra V, et al. Does preoperative platelet count and thrombocytosis play a prognostic role in patients undergoing nephrectomy for renal cell carcinoma? Results of a comprehensive retrospective series. World J Urol. 2013;31(5):1309-1316.

28. Vagdatli E, Gounari E, Lazaridou E, Katsibourlia E, Tsikopoulou F, Labrianou I. Platelet distribution width: a simple, practical and specific marker of activation of coagulation. Hippokratia. 2010;14(1):28-32.

29. Korniluk A, Koper-Lenkiewicz OM, Kamińska J, Kemona H, Dymicka-Piekarska V. Mean Platelet Volume (MPV): New Perspectives for an Old Marker in the Course and Prognosis of Inflammatory Conditions. Mediators Inflamm. 2019;2019:9213074.

30. Seles M, Posch F, Pichler GP, et al. Blood Platelet Volume Represents a Novel Prognostic Factor in Patients with Nonmetastatic Renal Cell Carcinoma and Improves the Predictive Ability of Established Prognostic Scores. J Urol. 2017;198(6):1247-1252.

31. Li M, Bai J, Wang S, et al. Mean platelet volume and platelet distribution width serve as prognostic biomarkers in skull base chordoma: a retrospective study. BMC Cancer. 2020;20(1):988.

32. Zuo $\mathrm{X}$, Kong $\mathrm{W}$, Feng $\mathrm{L}$, Zhang $\mathrm{H}$, Meng $\mathrm{X}$, Chen $\mathrm{W}$. Elevated platelet distribution width predicts poor prognosis in hepatocellular carcinoma. Cancer Biomark. 2019;24(3):307-313.

33. Huang Y, Cui MM, Huang YX, et al. Preoperative platelet distribution width predicts breast cancer survival. Cancer Biomark. 2018;23(2):205-211.

34. Kawakita Y, Motoyama S, Sato Y, et al. Prognostic Significance of Combined Platelet Distribution Width and C-Reactive Protein Score in Esophageal Cancer. Anticancer Res. 2020;40(10):5715-5725.

35. Cheng S, Han F, Wang Y, et al. The red distribution width and the platelet distribution width as prognostic predictors in gastric cancer. BMC Gastroenterol. 2017;17(1):163.

36. Chen $\mathrm{H}, \mathrm{Wu} \mathrm{Q}$, Zhang $\mathrm{Y}$, et al. Nomograms based on the novel platelet index score predict postoperative prognosis in endometrial cancer. Gynecol Oncol.. 2020;158(3):689-697. 